\title{
Word Identification for Mandarin Chinese Sentences
}

\author{
Keh-Jiann Chen Sling-Huan Liu \\ Institute of Information Science \\ Academia Sinica
}

\begin{abstract}
Chinese sentences are composed with string of characters without blanks to mark words. However the basic unit for sentence parsing and understanding is word. Therefore the first step of processing Chinese sentences is to identify the words. The difficulties of identifying words include (1) the identification of complex words, such as Determinative-Mcasurc, reduplications, derived words etc., (2) the identification of proper names,(3) resolving the ambiguous segmentations. In this paper, we propose the possible solutions for the above difficulties. We adopt a matching algorithm with 6 different heuristic rules to resolve the ambiguities and achieve an $99.77 \%$ of the success rate. The statistical data supports that the maximal matching algorithm is the most effective heuristics.
\end{abstract}

\section{Introduction}

Chinese sentences arc composed with string of characters without blanks to mark words. However the basic unit for sentence parsing and understanding is word. Therefore the first step of processing Chinese sentences is to identify the words( i.e. segment the character strings of the sentences into word strings).

Most of the current Chinese natural language processing systems include a processor for word identification. Also there are many word segmentation techniques been developed. Usually they use a lexicon with a large set of entries to match input sentences $[2,10,12,13,14,21]$. It is very often that there are many possible different successful matchings. Therefore the major focus for word identification were on the resolution of ambiguities. However many other important aspects, such as what should be done, in what depth and what are considered to be the correct identifications were totally ignored. High identification rates are claimed to be achieved, but none of them were neatsured under equal bases. There is no agrecment in what extend words are considered to be correctly identified. For instance, compounds occur very often in Chinese text, but none of the cxisting systems except ours pay much attention to identify them. Proper name is another type of words which cannot be listed exhaustively in the lexicon. 'Therefore simple matching algorithms can not successfully identify either compounds or proper names. In this paper, we like to raise the problems and the difficulties in identifying words and suggest the possible solutions.

\section{Difficulties in the Identification of Words}

As we mentioned in the previous chapter, the basic technique to identily the words is by matching algorithms. It requires a well prepared lexicon with sufficient amount of lexical entries which covers all of the Chinese words. However such a large lexicon is never existing nor will be composed, since the set of words is open ended. Not only because the new words will be generated but because there are unlimited number of compounds. Most of the word identification systems deliberately ignore the problem of compounds and leave the problem unsolved until the stage of parsing. We don't agree their view points and believe that different type of compounds should be handled by the different methods at different stages. Some types of the compounds had better to be handled before parsing, for they require different grammatical representations and identification strategies compared with the parsing of phrase structures. On the contrary, if the notphological rules for compounds have the same representation as the phrase structure rules, it is better to be identified at parsing stage. We will discuss this issue in more details in the later sections.

The other problem is that ambiguous segmentations frequently occur during the processing of word matching. It is because that very often a multisyllabic word contains monosyllabic words as its components. We have to try the different strategies to resolve such ambiguitics.

Many problems need to be solved, but first of all a lexicon should be composed for the matching algorithm.

\subsection{What are the Set of Words}

According to Liang's [14,15] definition, word is a smallest, mestningful, and frecly used unit. It is the basic processing unit for Chinese natural language processing. Since there is no morphological features as word segmentation marks, we have to adopt such a vague definition of the Chinese word. Liang [15] also propose a word scgmentation standard. However some of his view points are debatable and self contradictory. In fact it is almost impossible to define a standard for correct identification. 'Therefore instead of proposing a 
standard, we propose a criterion which should be followed by a good word segmentation algorithm. It is that a good segmentation algorithm should be able to produce a restilt which is suitable and sufficient for the purpose of later processing, such as parsing and understanding.

The set of words is open ended. Therefore the existing lexicons contain lexical entries which vary from 40 to 120 thousands. A large lexicon usually includes many compounds as well as many proper names, for it is hard to distinguish a word and a compound. For systems with a small lexicon, they might not perform worse than systems with a large lexicon, if they incorporate algorithms to identify compounds and proper names. However on the other hand there is no harm to have a large lexicon, once there is a way of handling the ambiguities, since statistically a large lexicon has the better chance to match words as well as producing ambiguous segmentations. Therefore we have the follow principle to collect the word set for the purpose of word segmentation.

Principle for composing a lexicon:

The lexicon should contain as many as possible words. If there is a doubt whether a string of characters is a word or a compound, you can just collect it as an entry.

Currently we have a lexicon of around 90 thousands entries, and keep updating for new words. A lexicon with such a size of course would still leave out many compounds and proper names. We use this lexicon to match the Chinese text, the result of the algorithm is a sequence of words defined in the lexicon. (1) is an instance of result.

\section{(1)}

a. jieshuoyuan Jau Shian-Ting yindau tamen interpreter Jau Shian-Ting guide them "The interpreter Shian-Ting Jau guided them."

b. pieshuo-yuan-Jau-Shian-'Ting-yindau-tamen

However we can see that some of the compounds and proper names are not identified as shown in (1)b. They were segmented into words or characters. Therefore at later stage those pieces of segments should be regrouped into compounds and proper names. We will discuss the issue at next two sections.

\subsection{Compounds}

There are many different type of compounds in Chinese and should be handled differently $[3,6,7,11$, $17,19]$.

a. determinative-measure compounds (IM)

A determinative-measure compound is composed of one or more determinatives, together with an oplional measure.

(2) je sari ben this three $C$ L "these three"

It is used to determine the reference or the quantity of the noun phrase that co-(xcurs with it. Despite the fact that both categories of determinatives and measures are closed, the combinations of them are not. However the set of DMs is a regular language which can be expressed by regular expressions and recognized by finite automata [19]. Mo [19] also point out that the structure of IOMs are exocentric. They are hardly similar to other phrase structures which are endocentric and context-free and can be analyzed by head driven parsing strategies. 'Therefore we suggest that the identification of DMs should be done in parallel with the identification of common words. There are 76 rules for DMs which covers almost all of the DMs [19]. For word identification, those rules function as a supplement for the lexicon, which works as if the lexicon contains all of the DMs. We will show the test result in the section 3.3.

b. Reduplications

In Chinese many verbs can be reduplicated to denote an additional meaning of trying the actions gently and relaxedly(3)[7].

(3) tiau tiau wu
jump jump dance
"dance a little"

This kind of morphological construction will not change the argument structure of the verbs, but do change their syntactic behavior. For instance, the reduplications can not cooccur with the adjuncts of postverbal location, aspect marker, duration, and quantifier [17]. In [17], they derived 12 different reduplication rules which cover the reduplication construction of verbs. In addition, there are 3 rules for the reduplication of DMs and 5 rules for A-not-A questions formation.

The identification of the reduplication construction should be done after the words have been identified, since it is better to see the words and then check whether part of the words has been reduplicated. It is a kind of context dependent process, so a separated process other than the process for DMs or Phrase structures, should be incorporated. 


\section{c. $A-n o t-A$ construction}

A-not-A constructions are commonly used in Chinese to form a question [3,7]. As we mentioned before, for $A-$ not-A construction, there are 5 different rules for reduplicating part of the verbs and coverbs $[17]$.

(4) fang-bu- fangshin

put not stop-worry

"stop worrying or not"

A-not-A construction is a kind of reduplication constrution. 'Therefore the technique for the identification of reduplication is also applicable for the identification of the A-Ilot-A construction.

\section{d. Derived Words}

A derived word is a compound which composed with a word of sten and a prefix or a suftix [11]. Derivative affixes are very productive in Mandarin Chinese.

(5) difang-shing

place quality

"localityr"

Those affixes usually are bound morphemes. In [11], we collect a set of most frequently occurred atfixes and study their morphological belaviors. We found that there are syntactic and semantic restrictions between modifiers and heads. Such a morphological patterns can be represented in terms ol Informationbased Case Grammar[5], which is also the grammatical formalism adopted for representing (hinese phrase structures in our parsing system [5,11]. Jollowing is an example of representation.

(6) shing Semantic:meaning: cquivalent of "-NESS", "-ITY", for expressing abstract notation

Syntactic: category: Nad leature: bound; $[+\mathrm{N},-\mathrm{V}]$

constraints: MK: $\{$ Vh1, V| transitive J, N $\}<<*$

Since the grammatical representation of derived words is the same as the representation of phrase structures, we suggest that the identification of the clerived words are better to be done at the parsing stage. Furthermore, the boundaries of the denived words are: syntactically ambiguous. They can not be identified without checking the contextual information.

\subsection{Proper Names}

Proper names occur very frequently in all kinds of articles. The identification of proper narnes becone one of the most difficult problems in Chinese natural language processing, since we can not exhaustly list all of the proper rame in the lexicon. Also there is no morphological nor punctuation makers to denote a proper name. Besides that a proper nane may contain a sub. string of common words. It makes the identification of the proper rames even harder. The only clue might be uscful in identilying proper nanes is the occurrences of bound moiphemes. Usually each Chinese character is a meaningful unit. Some of them can be used frecly as a word. Some are not; they have to be combined with other characters to form a word. Such characters can not be used freely as words, are named bound nor. phemes. If bound morphemes occur after word matching process, it means that there are derived words or proper nanes occurred in the text and have not been identified. The semantic classification of morphemes can be utilized to identify the different type of proper names. For instance, in [1], the set of sumames were used as a cluc to identify people's names and titles. 'There is no general solutions so far to handle the different types of proper mames. The only suggestion is that mark the proper nanes before identification process on treat the unknown strings as proper names.

\subsection{Ambiguities}

For Chinese claracter strings, they might have many dilferent well formed segmentations, but usually there is only onc granmatically and semantically sound segmentation for each sentence (7).

(7) yijing jenglichu jicguo alteady arrange- (sut result "Ihe result has come out." yijing-[jengli-chti]-jieguo yijing-[jeng-lichu]-jicguo

Therefore many algorithus; were proposed and heuristic or statistical preference rules were adopted for resolving anbiguities. However none of those rules has been thoroughly tested and provided their suceess rates. In the next section, we will state our algorithm as well as the heuristic rules and also provides the experiutent results in section 3.2 to show the success rate of cacli individuat rulc:

\section{Wond Identification Algorithiı}

According to the discussion of the chapter 2 , the picture of the word ictentification algorithm should be clearly as follows.

(8)

Ii lact not alt of the above processes were thorm onghly studies, but more or less some of them were studied and have successful results $[2,8,12,13,14,16$, $19,20,211$. Our word identification system adopt the alove sequence of algorithms, but we defer the second last process of finding derived words until parsing stage and the last process of finding proper mances is tempo- 


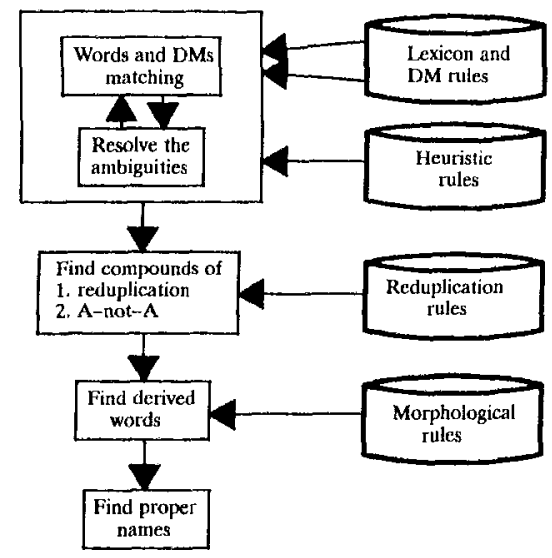

rary ignored for not having a feasible identification algorithm.

\subsection{Matching algorithm and disambiguation rules}

The first two steps of word identification algorithm are the word matching and disambiguation. These two processes were performed in parallel. Once an ambiguous match occurs, the disambiguation process is invoked immediately. The algorithm reads the input sentences from left to right. Then match the input character string with lexemes as well as DMs rules. If an ambiguous segmentation do occur, then the matching algorithm looks ahead two more words, then apply the disambiguation rules for those three word chunks. For instance, in (9), the first matched word could be 'wan' or 'wancheng'. Then the algorithm will look ahead to take all of the possible combinations of three word chunks, as shown in (10), into consideration.

(9) wanchengjianding baugau complete authenticate report "complete the report about authenticating"

(10) wan-cheng-jianding wancheng-jianding-bau wancheng-jianding baugau

The disambiguation algorithm will select the first word of the most plausible chunk as the solution. In this case, it is the word 'wancheng'. The algorithm then proceeds to process the next word until all the input text been processed.

The most powerful and commonly used disambiguation rule is the heuristic of maximal matching [12, $13,14,21]$. There are a few variations of the sense of maximal matching, but after we have done the cxperiments with each of different variations, we adopt the following maximal matching rules.

Heuristic rulc 1:

The most plausible segmentation is the three word sequence with the maximal length.

This heuristic rules achieves as high as $99.69 \%$ accuracy and $93.21 \%$ of the ambiguities were resolved by this rule. We will see the detail statistics in the next section. However there are still about $6.79 \%$ of ambiguities still can not be resolved by the maximal matching rule. Therefore we adopt the next heuristic rule.

Heuristic rule 2:

Pick the three word chunk which has the smallest standard deviation in the word length. This is equivalent to find the chunk with the minimal value on ( L(W1) - Mean) $* * 2+\left(\right.$ L(W2) - Mean) ${ }^{* *} 2+($ L(W3) Mean)**2, where $\mathrm{W} 1, \mathrm{~W} 2$, and $\mathrm{W} 3$ are threc words in a chunk; Mean is the average length of W1,W2, and W3; $L(W)$ denotes the length of the word $W$.

Heuristic rule 2 simply says that the word length are usually evenly distributed. For instance in (11), the segmentation of (11a) has the value 0 , but (11b) has value 2 . Therefore according to the heurist ic rule number 2 , the (11a) will be the selected solution and it is the correct segmentation.

(11) yianjiou shengmin chiyuan research life origin "to investigate the origin of life"
a. [yianjiou-shengmin]-chiyuan

b. [yianjiousheng-min]-chiyuan

However it may happen that there are more than two chunks with the same length and variance, we need a further resolution.

Heuristic rule 3:

Pick the chunk with fewer bound morphemes.

Heuristic rule 4:

Pick the chunk with fewer characters in DMs.

That is to say the normal words get higher priority than the bound morphernes and DMs. For instances examples $(12,13)$ were resolved by the rule 3 and 4 respectively. (12a) and (13a) are right choices.

(12) shietiau shang shoushiu jiau mafan negotiate up procedure more trouble- 
cated."

"In negotiation, the process is more compli-

a. shietiau-[shang-shoushiu]-jiau-mafan

b. shictiau-[shangshou-shiu]-jiau-mafan

(13) ta benren

he self

"he himself"

a. ta-benren

b. taben-ren

The heuristic rules 2,3 , and 4 only resolve $1.71 \%$ of the ambiguities as shown in the table 2 of the next section. After we observe the remaining ambiguities we found that many ambiguities were occurred due to the occurrences of monosyllabic words. For instances, the character string in (14a) can be segmented as (14b) or (14c), but none of the above resolution rules can resolve this case.

(14) ganran de chiuanshrrenshu shieshialai infect $\mathrm{DE}$ real number write

down

"write down the precise number of the in-

fected"

ganran-[de-chiuanshr]-renshu-shieshialai

ganran-[dechiuan-shr]-renshu-shieshialai

If we compare the correct segmentations with the incorrect segmentations, we find out that almost all of the monosyllabic word in the correct answer are function words, such as prepositions,conjunctions, as well as a few high frequent adverbs. And that the monosyllabic words in the incorrect segmentations are lower frequency words. The set of such frequently occurred monosyllabic words are shown in appendix 1 . We then have the following heuristic rule.

Heuristic rule 5 :

Pick the chunk with the high frequently occurred monosyllabic words.

This rule contributes $3.46 \%$ of the success of the ambiguity resolution. The remaining unsolved ambiguities are about $1.62 \%$ of the total input words. They usually should be resolved by applying real world knowledge or by checking grammatical validity. However it is almost impossible to apply real world knowledge nor to check the grammatical validity at this stage, so applying Markov model is a possible solution[21]. The other solution is much simpler, i.e. to pick the chunk with the highest accumulated frequency of words[22]. It requires the frequency counts for each words only instead of word bigram or trigram which required by the Markov model.

Heuristic rule 6:

Pick the chunk with the highest probability value. 'The probability value of the sequence of words ' W1 W2 W3' can be estimated by either

a) Markov model with the bigram approximation $\mathrm{P}=\mathrm{P}(\mathbf{W} 0 \mid \mathbf{W} 1) * \mathrm{P}(\mathrm{W} 1 \mid \mathbf{W} 2) * \mathrm{P}(\mathbf{W} 2 \mid \mathbf{W} 3)$ * P(W3) ; or

b) Word probability accumulation $\mathbf{P}=\mathbf{P}(\mathbf{W} 1)+\mathbf{P}(\mathbf{W} 2)+\mathbf{P}(\mathbf{W} 3)$

Heuristic rule Ga might not be feasible, since it re quires word bigram a matrix of size in the order of $10^{* *} 10$. But heuristic $6 \mathrm{~b}$ ) might not produce a satisfactory resolution. According to our experiment the success rate for $6 \mathrm{~b}$ ) is less than $70 \%$. Therefore the other solution is to retain the ambiguities and resolve at the parsing stage.

\subsection{Experiment Results}

We designed a word identification system to test the matching algorithm and the above mentioned heuristic rules. The lexicon for our system has about 90 thousands lexical entries plus unlimited amount of the DMs generated from 76 regular expressions. The 90 thousands lexemes form a word tree data structure in order to speed up the word matching $[4,10]$.For the same reasons, DM rules are compiled first to produce a Chomsky Normal Form like parsing table. The parsing table will then be interpreted during the word matching stage[19]. Two sets of test data are randomly selected from a Chinese corpus of 20 million characters. We summarize the testing result in Table 1 . Table 2 shows the success rates and applied rates for each heuristic rule.

The recall rate and recognition rate in the above table are defined as follows. Let

$\mathrm{N} 1=$ the number of words in the input text,

$\mathrm{N} 2=$ the number of words segmented by the system for the input text.

$\mathrm{N} 3=$ the number of words were correctly identified. Then the recall ratc is defined on be N3/N1 and the precision rate is $\mathrm{N} 3 / \mathrm{N} 2$. Ihe delinuluon of the other statistical result are chvously lioliowed the convention. The above testing algunthm du not include the process of handling derived wirds. Therefore the above statistics do not count the mistaken incurred due to the existence of derived worus. "r proper names.

We can see that the maxumal matching algorithm is the most effective heumstu. There are 10311 number of ambiguitics out of 17444 ikcurrences of the seg- 
mentations. It counts $58.94 \%$ of the total segmentations and $93.21 \%$ of ambiguities were resolved by this heuristics.

\section{Discussions and Concluding Remarks}

From the statistical results shown in table 2, it is clear that the maximal matching algorithm is the most useful heuristic method. Most of the mistakes caused by this heuristic are due to the occurrences of the words which are composed by two subwords. Those words are needed to have further investigations. If we want to further improve our system's performance, it seems that employing lexically dependent rules is unavoidable.
The crrors caused by the heuristic rule 2 are duc to the cases of a three character word followed by a monosyllabic word and which can be divided into two bisyllabic words, for instance (15).

(15) tzai shia san jou at down three week "in the following three week"

\section{[tzai-shiasanjou]} [tzaishia-sanjou]

Such mistakes can be avoided by giving the second bisyllabic words a lexically dependent marker which denotes that a low priority is given to this word when the heuristic rule 2 is applied.

Table 1. Testing results

\begin{tabular}{|l|r|r|r|}
\hline & Sample 1 & Sample 2 & Total \\
\hline of sentences & 833 & 1968 & 2801 \\
\hline of characters & 8455 & 20879 & 29334 \\
\hline of wonds & 5085 & 12409 & 17494 \\
\hline by the system & 5076 & 12399 & 17475 \\
\hline $\begin{array}{l}\text { identifications } \\
\text { recall rate }\end{array}$ & 5064 & 12370 & 17434 \\
\hline precision rate & $99.58 \%$ & $99.69 \%$ & $99.66 \%$ \\
\hline
\end{tabular}

Table 2. The success rates of the heuristic rules

\begin{tabular}{|c|c|c|c|c|c|c|c|c|c|}
\hline & \multicolumn{3}{|c|}{ Sumple 1} & \multicolumn{3}{|c|}{ Sumple 2} & \multicolumn{3}{|c|}{ Total } \\
\hline & of identifications & I of exrors & success rate & I of inentifications & of errors & success rate & of ilentification & I of errors & sticcess rate \\
\hline Heuriatic & & & & & & & & & \\
\hline Rule 1 & 2875 & 13 & $99.55 \%$ & 6938 & 17 & $99.75 \%$ & 9813 & 30 & $99.67 \%$ \\
\hline $\begin{array}{l}\text { Heariatiof } \\
\text { Rule } 2\end{array}$ & 36 & 4 & $88.89 \%$ & 74 & 3 & $95.95 \%$ & 110 & 7 & $93.64 \%$ \\
\hline $\begin{array}{l}\text { Heurintio } \\
\text { Rule I }\end{array}$ & 0 & 0 & $100 \%$ & 5 & 0 & $100 \%$ & 5 & 0 & $100 \%$ \\
\hline $\begin{array}{l}\text { Eleurintio } \\
\text { Rule } 4\end{array}$ & 18 & 0 & $100 \%$ & 32 & 1 & $96.86 \%$ & 50 & 1 & $98,00 \%$ \\
\hline $\begin{array}{l}\text { Heuriatid } \\
\text { pules }\end{array}$ & 104 & 2 & $98.08 \%$ & 238 & 12 & $94.96 \%$ & 342 & 14 & $95.90 \%$ \\
\hline $\begin{array}{l}\text { Heuriatic } \\
\text { Rule } 6\end{array}$ & 48 & 15 & $68.75 \%$ & 109 & 36 & $66.97 \%$ & 157 & 51 & $67.52 \%$ \\
\hline
\end{tabular}


The heuristic rules $\# 3$ and $\# 4$ are the most reliable disambiguation rules. However they only contribute $0.53 \%$ of the disambiguation processes.

The heuristic rule $\# 5$ is useful, but the priority values for each ligh frequent monosyllabic word has to be carefully rearranged in order to reduce possible mistakes [18].

The heuristic rule $\# 6$ needs to be further studied. It will be much more casier to use the bigram or trigram based on grammatical categorics instead of the word bigram or the simple accumulation of the word frequencies. It will be the future study.

About the identification of the proper names, it requires a further investigation on the results of the proper names after segmentation algorithm is applied.

\section{References}

[1] J. S. Chang, "A Multiple-Corpus Approach to Identi cation of Chinese Surname--Names," Proc. of Natural Language Processing Pacific Rim Symposium, Singapore, 1991

[2] J. S. Chang, J. I. Chang and S. D. Chen, "A Method of Constraint Satisfaction and Statistical Optimization for Chinese Word Segmentation," Proc. of the 1991 R. O. C Computational Linguistics Conference, Taiwan, 1991

[3] Y. R. Chao, A Grammar of Spoken Chinese, University of Califomia Press, California, 1968

[4] K. J. Chen, C. J.Chen and L. I. Lee, "Analysis and Research in Chinese Sentences - Segmentation and Construction," 'Technical Report, TR-86-004, Nankang, Academia Sinica, 1986

[5] K. J. Chen and C. R. Hulang, "Infonnation-based Case Grammar," COLING - 90, Vol 2, p.54-p.59

[6] K. J. Chen et al, "Compounds and Parsing in Mandarin Chinesc," Proc. of National Computer Symposium, 1987

[7] G. Y. Chen, "A-not-A Questions in Chinese," manuscript, CKIP group, Academia Sinica, Taipei, 1991

[8] C. K. Fan and W. H. Isai, "Autonatic Word Identification in Chinese Sentences by the Relaxation Technique," Computer Processing of Chinese and Oriental Languages, Vol.4, No.1, November 1988

[9] R. Garside, G. Leech and (i. Sampson, "The Computational Analysis of English -- a Corpus- based Approach," Longman Group UK Limited, 1987

[10] W. H. Ho, "Automatic Recognition of Chinese Words," Master Thesis, National Taiwan Institute of Technology, Taipei, Taiwan, 1983

[11] W. M. Hong, C. R. Huang, T. Z. Tang and K. J. Chen," The Morphological Rules of Chinese Derivative Words, "Io be presented at the 1991 Inter. national Conference on Teaching Chinese as a Second Language, December, 1991, Thipei

[12] C. Y.Jic, Y. Liu and N. Y. Liang, "Oni Methods of Chinese Automatic Segmentation," Journal of Chirtese Information Processing, Vol.3, No.1, 1989

[13] H. I. Li, S. Lien, C. F. Sun and M. S. Sun, "A Maximal Matching Automatic Chinese Word Segmentation Algorithm Using Corpus Tagging for Ambiguity Resolution," Proc. of the 1991 R. O. C Computational Linguistics Conference, Taiwan, 1991

[14] N. Y. Liang, "Autonatic Chinese Text Word Segmentation System - CDWS", Journal of Chinese Information Processing, Vol.1, No.2, 1987

[15] N. Y. Liang, "Contemporary Chinese Language Word Segmentation Standard Used for Information Processing," 1989, a draft proposal

[16] N. Y. Liang, "The Knowledge of Chinese Words Segmentation," Journal of Chinese Information Processing, Vol.4, No.2, 1990

[17] M. L. Lin, "The Grammatical and Semantic Propcrties of Reduplications," manuscript, CKIP" group, Academia Sinica, 1991

[18] I. M. Liu, C. Z. Chang and S. C. Wang, "Frequency Count of Frequently Used Chinese Words," Taipei, Tàiwan, Lucky Book Co., 1975

[19] K. P. Mo, Y. J. Yang, K. J. Chen and C. R. Huang, "Determinative-Measure Compounds in Mandarin Chinese : Their Formation Rules and Parser Implementation," Proc. of the 1991 R.O.C Computational Linguistics Conference, làiwan, 1991

[20] R. Sproat and C. Shih, "A Statistical Method for Funding Word Boundaries in Chinese 'Text," Computer Processing of Chinese and Oriental Languages, Vol.4, No.4, March $19 \%$

[21] C. L. Yeh and H. J. Lee, "Rule-based Word Identification for Mandarin Chinese Sentences -- A Unification Approach," Computer Processing of Chinese and Oriental Languages, Vol.5, No.2, March 1991 